\title{
A High-Resolution Amperometric Acetylcholine Sensor Based on Nano- Assembled Carbon Nanotube and Acetylcholinesterase Thin Films
}

\author{
Wei Xue ${ }^{a}$ and Tianhong Cuib \\ Department of Mechanical Engineering, University of Minnesota \\ 111 Church Street S.E., Minneapolis, MN 55455, USA \\ axuewei@me.umn.edu, btcui@me.umn.edu (corresponding author)
}

Received February 2, 2007; received in revised form May 1, 2007; accepted May 8, 2007

\begin{abstract}
Keywords: Carbon nanotube; Acetylcholine; Acetylcholinesterase; Layer-by-layer self-assembly; Thin film; Sensor.
\end{abstract}

\begin{abstract}
We demonstrate a carbon nanotube based high-resolution biosensor for acetylcholine sensing. Carbon nanotubes are deposited on a silicon wafer in a repeated fashion with layer-by-layer nano self-assembly technique. With nano-assembled acetylcholinesterase molecules on the surface, the carbon nanotube biosensor is capable of detecting acetylcholine at an ultra-low concentration of $100 \mathrm{pM}$. The sensitivity of the acetylcholine sensor is measured as $7.2 \mu \mathrm{A} /$ decade. The real-time measurement shows the response time of the biosensor is approximately $6 \mathrm{sec}$. Both the carbon nanotube film and the acetylcholinesterase film are crucial in the sensing process. Due to its high resolution, fast response, small size, and low cost, the carbon nanotube biosensor has tremendous potential for applications in medical research and clinical diagnosis.
\end{abstract}

\section{Introduction}

Acetylcholine $(\mathrm{ACh})$, the first identified neurotransmitter, can be found in both peripheral and central nerve systems (PNS and CNS) in mammals including humans [1]. In the PNS, ACh binds to acetylcholine receptors (AChR) and regulates muscle contraction. In the CNS, ACh plays a crucial role in the processes related to behavioral activities, arousal, attention, learning, memory, etc. ACh is synthesized in neurons from choline by means of choline acetyltransferase (ChAT) and acetylcoenzyme A [2]. The ACh concentration in human blood is approximately $8.66 \pm 1.02 \mathrm{nM}$ [3]. The dysfunctional $\mathrm{ACh}$ regulation in the brain causes neuropsychiatric disorders such as Parkinson disease, Alzheimer disease, and myasthenia gravis. In order to study the illnesses and develop the medicines, it is important to measure the ACh concentration with simple, fast, inexpensive, and accurate methods. Common techniques for ACh detection include high-performance liquid chromatography (HPLC) method on microdialysis samples [4], electrochemical methods using potentiometric and amperometric sensors [3,5], and ion-sensitive field-effect transistors (ISFETs) [6]. HPLC on microdialysis samples has very high resolution in the $\mathrm{pM}$ range. However, this method suffers from tedious sample pretreatment and high cost; for example, a microdialysis system costs more than $\$ 25,000$, and a microdialysis probe costs about $\$ 200$. Electrochemical sensors and ISFETs for ACh detection have the advantages of low cost, small size, easy detection, and short response time. However, the resolution of these devices is relatively low, normally in the $100 \mathrm{nM}$ to $10 \mu \mathrm{M}$ range. A recent study used a $\mathrm{pH}$-sensitive poly(vinyl chloride) (PVC) membrane with a plasma-polymerized film as a potentiometric biosensor for ACh sensing, the resolution if this biosensor is $2 \mathrm{nM}$ and the detection reaches $100 \mu \mathrm{M}$ [1]. The performance of the device exceeds most reported ACh microsensors. However, the characteristic curve is not linear and calibration is required for this device.

The inherent properties such as large surface area and high conductivity make carbon nanotubes (CNTs) promising candidates for chemical- and bio-sensing applications. Pristine semiconducting single-walled carbon nanotubes (SWNTs) are sensitive to electron-donating (such as $\mathrm{NH}_{3}$ ) and electron-accepting (such as $\mathrm{NO}_{2}$ ) gas molecules [7]. CNTs with proper chemical and/or physical 
modifications are capable of detecting more types of molecules such as $\mathrm{H}_{2}, \mathrm{CO}$, glucose, and DNA $[8,9]$. A recent study demonstrates that CNT-modified electrodes can greatly enhance the performance of electrochemical amperometric biosensors [10]. CNTs can serve as the "reservoir" of the biomolecules. They can also accelerate the carrier transfer and minimize the surface fouling. In addition, CNTs have higher surface renewability than most materials. For example, the CNTmodified electrode can produce steady-state signals within 8-10 sec [11]. Different forms of SWNTs, including pristine SWNTs, SWNT composites, and SWNT thin films, can be used in the sensing applications. The SWNTs offer advantages over traditional sensor materials in terms of resolution, sensitivity, operation temperature, and dimension.

In this paper, we report a SWNT thin film based microelectronic device for ACh sensing. The sensor is a stand-alone device with a simple resistor structure. The two-terminal structure obviates the need for reference electrodes and electrolyte electron mediators, which are often required for the traditional electrochemical microsensors. Two gold electrodes are connected with a SWNT thin film. The SWNTs, in the form of a multilayer, are fabricated with layer-by-layer (LbL) nano selfassembly. LbL self-assembly is a simple, low-cost, and low-temperature technique with high controllability and versatility. Oppositely charged polyelectrolytes, nanoparticles, CNTs, and biomolecules can form well-organized multilayers in nanometer scale based on electrostatic force with this technique. Compared with other coating techniques for SWNT biosensors, self-assembly can precisely control the amount of SWNTs in the film. The multilayer film of the ACh sensor is composed of two parts. Alternating layers of SWNTs and poly(dimethyldiallylammonium chloride) (PDDA) work as the conducting component. Alternating layers of acetylcholinesterase (AChE) enzymes and poly(sodium 4-styrenesulfonate) (PSS) work as the sensing component. Both the SWNT film and the AChE film are crucial in the sensing process. The SWNT sensor shows a high resolution and short response time for ACh sensing. The measured resolution of $100 \mathrm{pM}$ is much higher than other reported ACh microsensors. With an optimized design, the SWNT sensor may achieve higher performance.

\section{Materials}

Aqueous polyelectrolyte solutions were obtained from Sigma-Aldrich and diluted with deionized (DI) water. The concentrations of PDDA (polycation) and PSS (polyanion) are $15 \mathrm{mg} / \mathrm{ml}$ and 3 $\mathrm{mg} / \mathrm{ml}$, respectively. $\mathrm{NaCl}(0.5 \mathrm{M})$ was added to both solutions to increase the ionic strength of the polyelectrolytes. ACh and AChE were also obtained from Sigma-Aldrich. AChE was diluted with DI water to $10 \mu \mathrm{g} / \mathrm{ml}$. $\mathrm{NaCl}(0.1 \mathrm{mM})$ was added to the AChE solution for ionic strength enhancement.

SWNTs were obtained from Chengdu Organic Chemical Co. Ltd. The SWNTs were treated with nitric and sulfuric acids $\left(1: 3 \mathrm{HNO}_{3}: \mathrm{H}_{2} \mathrm{SO}_{4}\right)$ at $110^{\circ} \mathrm{C}$ for $45 \mathrm{~min}$. The acid treatment can greatly enhance the water solubility of SWNTs by introducing carboxylic $(-\mathrm{COOH})$ functional groups to the sidewalls and ends. After the treatment, the SWNTs are negatively charged and uniformly dispersed in DI water with a concentration of approximately $1 \mathrm{mg} / \mathrm{ml}$.

\section{Fabrication}

Recently, we reported the structural characterization of the self-assembled SWNT multilayer films using a quartz crystal microbalance (QCM) [12]. The growth of the SWNTs is highly repeatable. The average thickness of a (PDDA/SWNT) bi-layer is approximately $7.6 \mathrm{~nm}$. The charge property of the AChE molecules can also be determined by the QCM [13]. The QCM characterization shows that AChE is a positively charged enzyme and can be assembled on the crystal in alternate with PSS, as shown in Fig. 1. The average frequency shifts for PSS and AChE are -66.7 and $-143.4 \mathrm{~Hz}$, respectively. Based on the Sauerbrey equation [12], the corresponding thicknesses for PSS and AChE are 3.1 and $6.1 \mathrm{~nm}$, respectively. Therefore, the thickness of a (PSS/AChE) bi-layer is 9.2 $\mathrm{nm}$. The saturation adsorption time for AChE is $15 \mathrm{~min}$. The self-assembly process for both SWNTs and $\mathrm{AChE}$ is done at room temperature. 


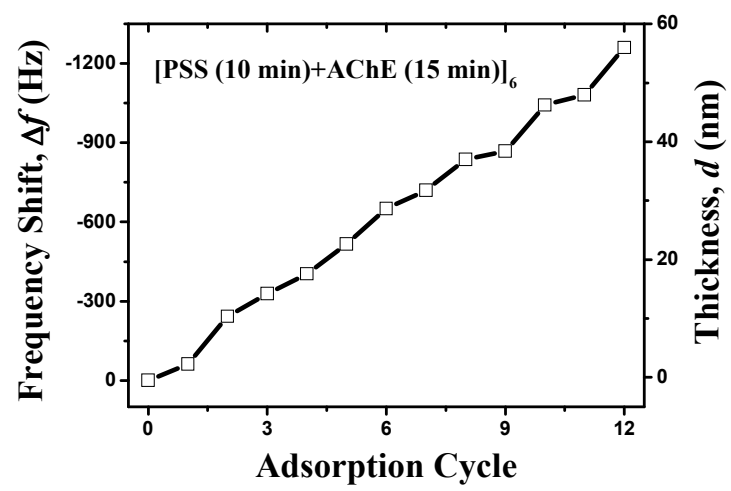

Fig. 1. Growth of a (PSS/AChE) 6 multilayer monitored with the QCM.

(a)

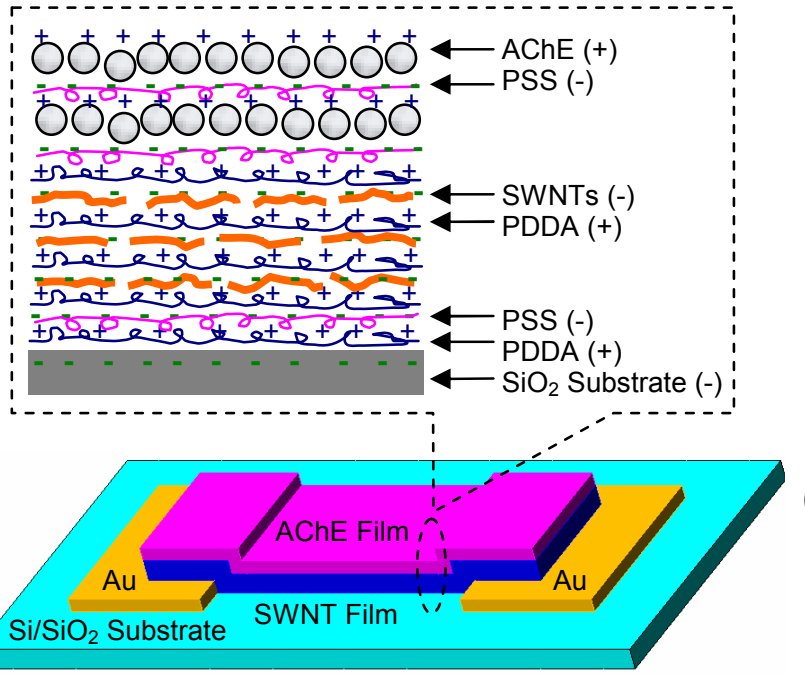

(b)
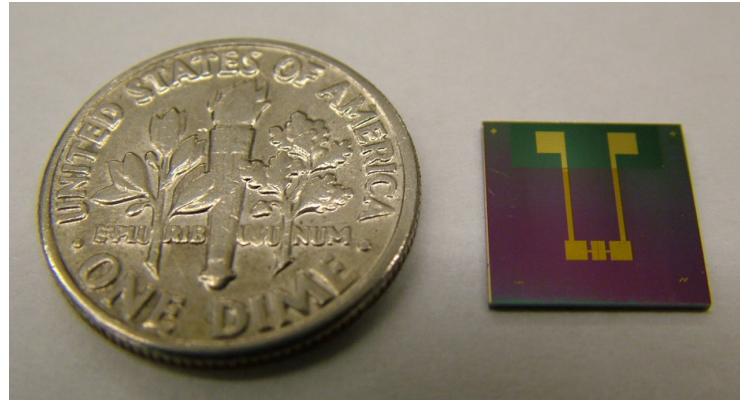

(c)

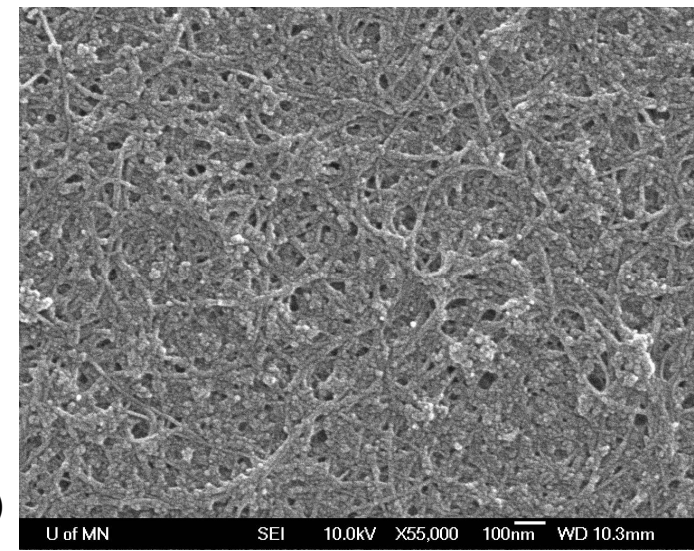

(d)

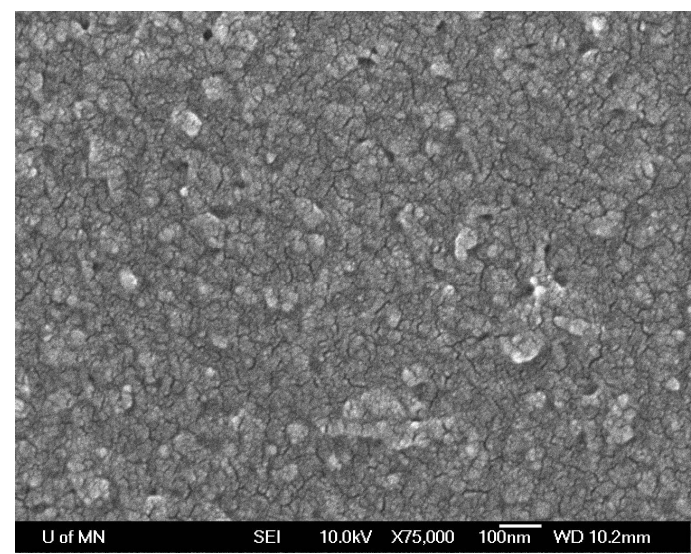

Fig. 2. (a) Structure of a SWNT thin-film ACh biosensor. Au is patterned as the electrodes on the surface of a $\mathrm{Si} / \mathrm{SiO}_{2}$ substrate. The channel between the Au electrodes is $5 \mu \mathrm{m}$ long and $1 \mathrm{~mm}$ wide. SWNT and ACh thin films are deposited on the substrate as the conducting film and the sensing film, respectively. The self-assembled films consist of a (PDDA/PSS) $)_{2}$ precursor film, a $(\mathrm{PDDA} / \mathrm{SWNT})_{5}$ conducting film, and a $(\mathrm{PSS} / \mathrm{AChE})_{3}$ sensing film. (b) Optical image of a fabricated device next to a dime and the close-up images of the channel area. (c) Scanning electron microscopy (SEM) image of the assembled SWNTs. (d) SEM image of assembled AChE.

Fig. 2a shows the structure of the SWNT thin-film biosensor and the construction of the multilayer films. The sensor is fabricated on a 4-inch silicon ( $\mathrm{Si}$ ) wafer with $300 \mathrm{~nm}$ silicon dioxide $\left(\mathrm{SiO}_{2}\right)$ layer on the surface. Chromium $(\mathrm{Cr}, 100 \mathrm{~nm}$, adhesion material) and gold (Au, $200 \mathrm{~nm}$, electrode material) are deposited on the wafer with electron-beam evaporation. A layer of positive 
photoresist (Shipley PR1813) is spin coated on the surface and patterned with UV lithography. The $\mathrm{Cr} / \mathrm{Au}$ layers are patterned as source and drain electrodes with wet etching technique. The etchants for etching $\mathrm{Cr}$ and $\mathrm{Au}$ are CR-12S (from Cyantek Co.) and GE-6 (from Transene Co.), respectively. After removing the remaining photoresist with acetone, another photoresist layer is spin coated on the substrate and patterned with UV lithography. The channel area (channel dimension: $5 \mu \mathrm{m} \times 1$ $\mathrm{mm}$ ) is exposed and the electrodes remain covered. The multilayer films are deposited on the substrate with LbL self-assembly process. First, the wafer is alternately immersed in PDDA and PSS solutions in a sequence of [PDDA $(10 \mathrm{~min})+$ PSS $(10 \mathrm{~min})]_{2}$. The two (PDDA/PSS) bi-layers are used to pre-charge the substrate and prepare a smooth base for the subsequent adsorption of carbon nanotubes [14]. Following the (PDDA/PSS) 2 precursor multilayer, five SWNT layers are coated on the substrate alternately with PDDA. The sequence of the alternate immersion is [PDDA $(10 \mathrm{~min})+\mathrm{SWNT}(15 \mathrm{~min})]_{5}$. The $(\mathrm{PDDA} / \mathrm{SWNT})_{5}$ multilayer provides a highly conductive channel for carrier transfer. The electrodes are uncovered with lift-off technique by soaking the substrate in acetone for $1 \mathrm{~min}$ with the assistance of ultrasonic vibration. Acetone dissolves the photoresist and removes the SWNT multilayer from those areas. The final step is to coat the AChE on top of the SWNT film as the sensing material using the LbL self-assembly. The sequence of the AChE deposition is PDDA (10 min $)+[\operatorname{PSS}(10 \mathrm{~min})+\operatorname{AChE}(15 \mathrm{~min})]_{3}$. The final nano-assembled films include a (PDDA/PSS $)_{2}$ precursor film, a (PDDA/SWNT) $)_{5}$ conducting film, and a $(\mathrm{PSS} / \mathrm{AChE})_{3}$ sensing film. From the QCM characterization, the thicknesses of the SWNT and AChE films can be estimated as 38 and $27.6 \mathrm{~nm}$, respectively.

Fig. $2 \mathrm{~b}$ shows an optical image of a fabricated device. The size of the fabricated device is $1 \mathrm{~cm} \times$ $1 \mathrm{~cm}$, which is smaller than a dime. The electrodes are elongated to the edge of the device for easy probing and detection. The scanning electron microscope (SEM) images of the assembled SWNT and AChE films are shown in Figs. 2c and 2d, respectively. The SWNTs are interconnected and form a dense network. Both films are relatively uniform due to the presence of the intermediate polyelectrolyte layers.

\section{Measurement}

The electrical measurement system setup is illustrated in Fig. 3a. The device is partially dipped in the ACh solution. The multilayer films on top of the conducting channel are immersed in the solution, and the elongated electrodes remain exposed in the air. A voltage, $V$, is applied on the sensor by attaching two probes to the electrodes. At the same time, the current flowing through the channel, $I$, is measured and recorded. All the measurements are done in aqueous solutions at room temperature.

The hydrolysis process of ACh is shown in Fig. 3b. ACh is hydrolyzed into choline and acetic acid in the presence of $\mathrm{AChE}$, and the generated acetic acid releases hydrogen ions $\left(\mathrm{H}^{+}\right)$to the solution. Fig. 3c shows the schematic sensing mechanism of the SWNT thin-film biosensor. The sensing process of the device consists of three steps. First, the ACh molecules in the solution diffuse into the nano-assembled thin film. Second, the AChE immobilized on the device surface catalyzes the enzyme reaction and releases hydrogen ions. The concentration of the released hydrogen ions in the local area including the film-liquid interface and inside the AChE film is determined by the concentration of ACh. Solutions with higher ACh concentration can generate more hydrogen ions. The concentration of the released hydrogen ions in the local area including the film-liquid interface and inside the AChE film is proportional to the concentration of the ACh in the solution. Therefore, a concentration gradient of the hydrogen ions is established in this area. Third, the hydrogen ions diffuse to the surrounding areas following the concentration gradient. Some hydrogen ions diffuse into the bulk ACh solution, while others diffuse into the inner SWNT conducting film. Early study shows that the pristine semiconducting SWNT is a p-type material; i.e., the majority carriers in the SWNTs are holes [15]. From our investigation, the acid-treated SWNTs also show p-type properties [16]. The diffused hydrogen ions join the holes in the SWNT conducting film and increase its conductance. The applied voltage drives all the positive charges across the source/drain electrodes. Due to the large surface area and high conductivity, the nano-assembled SWNTs provide a 
conductive channel for the hydrogen ions and holes. The increase of current represents the concentration of hydrogen ions in the SWNT film. In other words, the ACh concentration can be monitored and determined by measuring the current of the sensor.

(a)
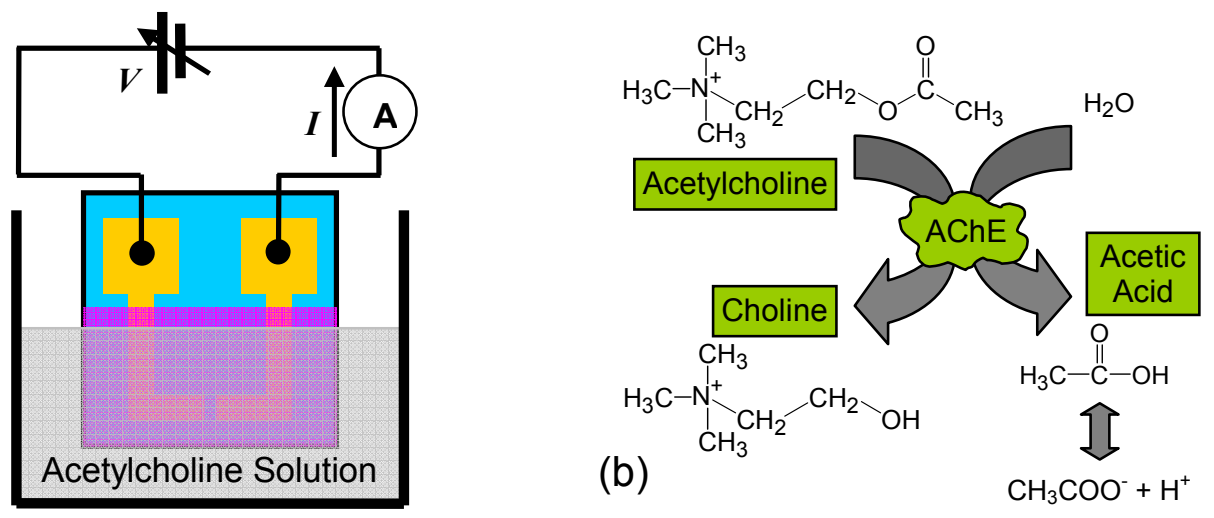

(c)

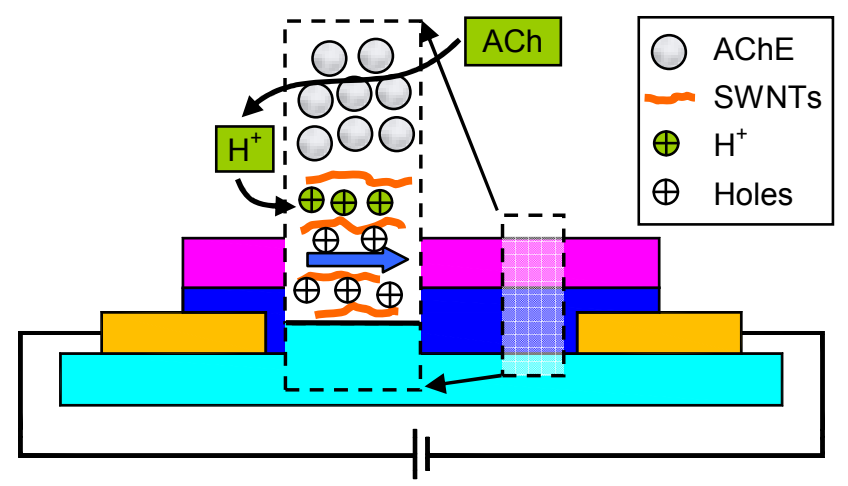

Fig. 3. (a) Measurement scheme for ACh sensing. (b) Hydrolysis of ACh in the presence of AChE. (c) Sensing mechanism of the SWNT thin-film ACh sensor. Hydrogen ions $\left(\mathrm{H}^{+}\right.$, dark dots) are released during the ACh hydrolysis process. The hydrogen ions join the holes (white dots) in the SWNT film for current transfer.

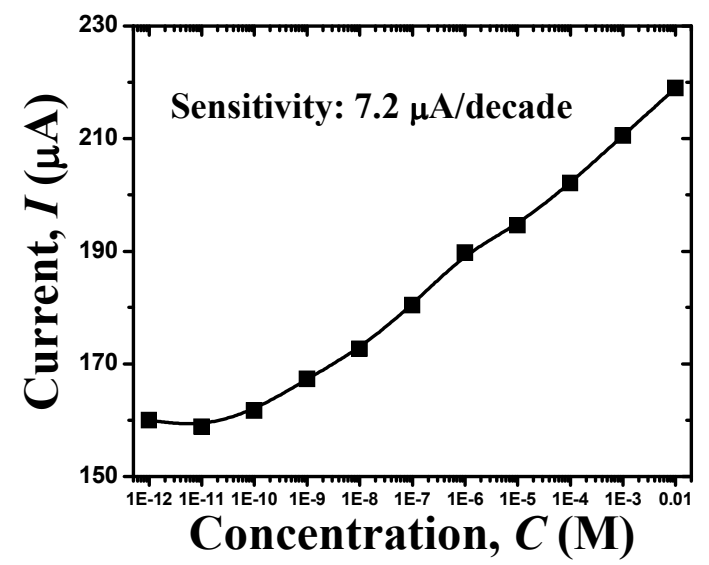

Fig. 4. Resolution and sensitivity measurement of ACh sensor. The voltage applied on the device is $750 \mathrm{mV}$. The scatters are the experimental data and the line is the fitted curve.

\section{Results and Discussion}

As mentioned above, the fabricated devices are measured in aqueous solutions at room temperature. The voltage $V$ across the electrodes is set as $750 \mathrm{mV}$. To allow the sensor to reach a steady state, the current is recorded after dipping the sensor in the solution for $1 \mathrm{~min}$. Between two measurements, 
the sensor is rinsed thoroughly with DI water. Fig. 4 shows the current values obtained at different ACh concentrations ranging from $1 \mathrm{pM}$ to $10 \mathrm{mM}$. At the low concentration, the detected current is approximately $160 \mu \mathrm{A}$. The current rises when dipping the sensor in the ACh solution with higher concentrations. The curve shows a linear relationship between the ACh concentration (in log scale) and the current at concentrations higher than $100 \mathrm{pM}$. The sensitivity of the device is obtained from the slope of the linear-region curve as approximately $7.2 \mu \mathrm{A} /$ decade. The measured resolution of this device is $100 \mathrm{pM}$, which is much higher than other reported ACh microsensors [3,5,6]. In order to verify the measured results, we fabricate a number of sensors with two different channel lengths. For devices with $5 \mu \mathrm{m}$ channels, the resolution is typically in between $100 \mathrm{pM}$ and $1 \mathrm{nM}$. For devices with $10 \mu \mathrm{m}$ channels, the resolution is slightly lower, normally in the $1 \mathrm{nM}$ to $10 \mathrm{nM}$ range. In the air environment, the current of the device is measured as $319.8 \mu \mathrm{A}$, higher than that in the aqueous solutions. The conductance of the device is therefore calculated as $426 \mu \mathrm{S}$.

(a)
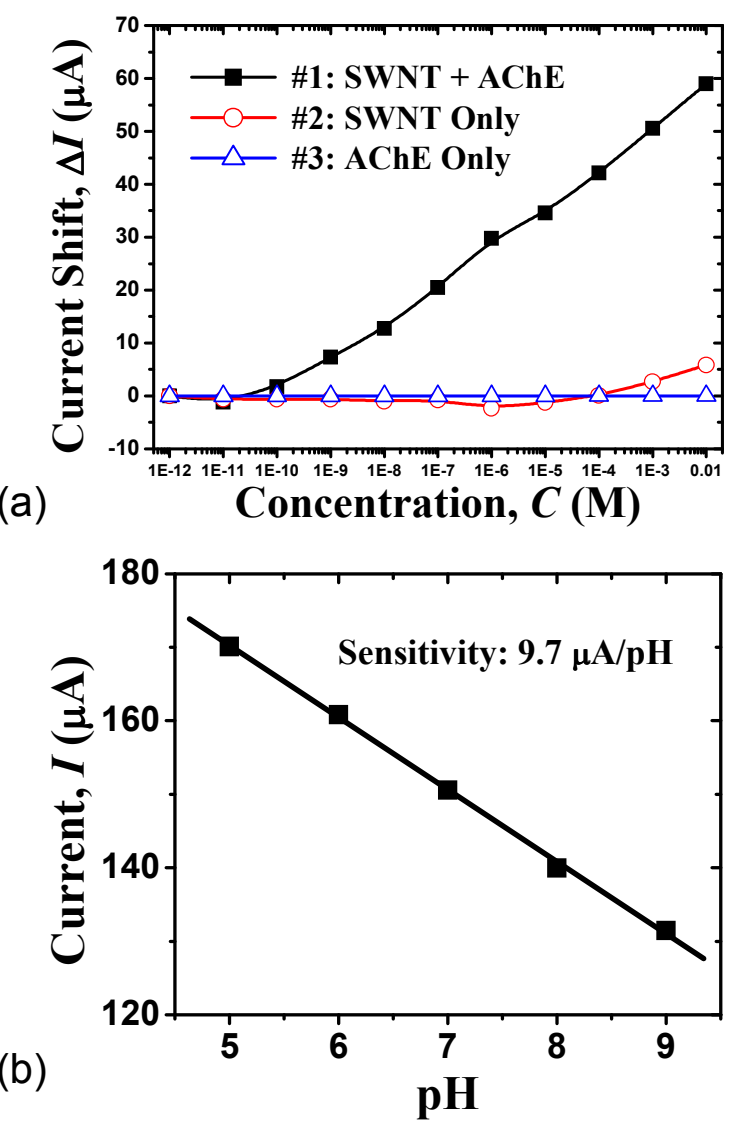

Fig. 5. (a) Resolution and sensitivity measurement of ACh sensors with different coatings. Sensor-1 is covered with both SWNT and AChE films (the data are from Fig. 4). Sensor-2 is covered with SWNT film. Sensor-3 is covered with AChE film. (b) Hydrogen ion sensitivity of sensor-2. The $\mathrm{pH}$ solutions are mixtures of $50 \mathrm{mM} \mathrm{KH_{2 }} \mathrm{PO}_{4}$ and $\mathrm{K}_{2} \mathrm{HPO}_{4}$ buffers. The voltages applied on the devices are $750 \mathrm{mV}$. For both figures, the scatters are the experimental data and the lines are the fitted curves.

To determine the roles of SWNT and AChE films, devices with different coatings are fabricated. All the devices have $5 \mu \mathrm{m}$ channels, and they are measured with the same system under the same condition. Fig. 5a compares the current shift data of three devices. For all the devices, the current detected in the $1 \mathrm{pM}$ ACh solution is set as 0 , a starting point. The curve of sensor- 1 is from Fig. 4 . This sensor is coated with both (PDDA/SWNT) $)_{5}$ and (PSS/AChE) $)_{3}$ multilayers. Sensor-2 is only coated with (PDDA/SWNT) $)_{5}$ multilayer and sensor-3 is only coated with (PSS/AChE) $)_{3}$ multilayer. Within the detection range $1 \mathrm{pM}-10 \mathrm{mM}$, the current shifts for three sensors are $59 \mu \mathrm{A}, 5.8 \mu \mathrm{A}$, 
and $28 \mathrm{nA}$, respectively. For sensor-2 and sensor-3, the currents remain almost unchanged in the low concentration region. Slight current increases can be observed at concentrations higher than 100 $\mu \mathrm{M}$. From this figure, both SWNT and AChE films are proved to be crucial in the sensing process. For the fabricated thin-film sensor, the outmost film is an AChE multilayer. The AChE multilayer can catalyze ACh and release hydrogen ions. However, it is also a barrier to impede the diffusion of ACh and hydrogen ions into the SWNT conducting film. To determine the effect of the outmost AChE multilayer, we use sensor-2 (a device without AChE multilayer) to detect $\mathrm{pH}$ solutions, as

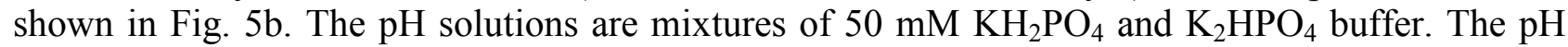
values range from 5 to 9 . The sensor shows a linear $\mathrm{pH}$ response, and the sensitivity is calculated as $9.7 \mu \mathrm{A} / \mathrm{pH}$. Compared with the sensitivity obtained for ACh sensor $(7.2 \mu \mathrm{A} /$ decade $)$, the outmost AChE multilayer decreases the sensitivity of the device for approximately $26 \%$.

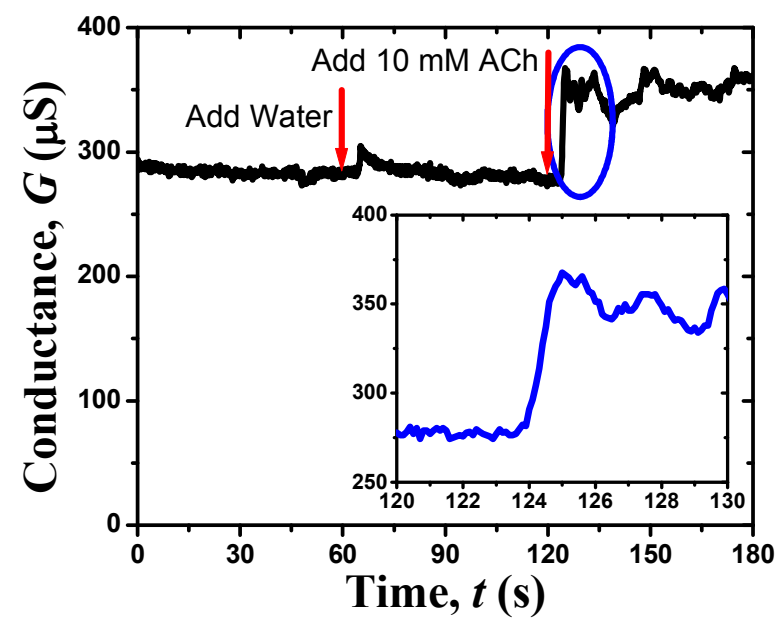

Fig. 6. Time-dependent response of the SWNT thin-film sensor to DI water and ACh solution. The channel of the sensor is initially covered with $500 \mu \mathrm{l}$ DI water. After $60 \mathrm{sec}, 500 \mu \mathrm{l}$ DI water (left arrow) is injected to the solution, and after $120 \mathrm{sec}, 1 \mathrm{ml} 10 \mathrm{mM} \mathrm{ACh}$ solution (right arrow) is injected to the solution. The conductance response (oval) of the device is enlarged and shown as an inset. The sensor reaches a steady state within $6 \mathrm{sec}$.

The time-dependent response of the SWNT thin-film sensor is recorded by a data acquisition system and shown in Fig. 6. The measurement starts with covering the channel of the device with $500 \mu \mathrm{DI}$ water. The initial conductance of the device in DI water is approximately $284 \mu \mathrm{S}$. When adding another $500 \mu \mathrm{l}$ DI water to the solution, the transient conductance increases first and then returns to its initial value. When adding $1 \mathrm{ml} 10 \mathrm{mM}$ ACh solution to the solution, an obvious conductance increase can be observed, and the final conductance is approximately $360 \mu \mathrm{S}$. A delay of $4 \mathrm{sec}$ can be observed in both cases. It is caused by the manual operation error and the fluid diffusion. An enlarged view of the response is shown in the same figure as an inset. The conductance reaches a steady state within $6 \mathrm{sec}$. However, transient overshoot can be observed from the curve when adding DI water and ACh solution. The transient conductance changes are caused by the injection of liquid. Even though the liquid is injected slowly, the flow has impact on the SWNT thin film. The nano-assembled SWNT film can slightly change its construction during the liquid injection, for example, the polyelectrolyte molecules and carbon nanotubes expand in the aqueous solution because the same charges on the chain repel each other [17], and the injected flow can impulse them and change their positions. After reaching the steady state in $6 \mathrm{sec}$, the SWNT thin film structure reaches an equilibrium status and the conductance of the SWNT thin film remains relatively stable afterwards.

The fabricated SWNT thin-film sensor shows a high resolution and a fast response. To further improve the performance, there are several parameters can be modified and optimized. First, the structure of the sensing film can be modified. Our current design uses a top AChE sensing film to 
cover the sensor. The fully covered AChE film is able to generate more hydrogen ions but it also prevents the diffusion of them into the SWNT conducting film. Because the performance of the sensor is mainly determined by the generation and transport of the hydrogen ions, an optimized design can result in a more efficient transport of the ions. For example, an interdigital finger design of the AChE film may provide a more effective diffusion of the hydrogen ions into the SWNT film. For the same reason, an optimized number of AChE layers may increase the performance of the device. Second, the channel length can be changed. As described above, $5 \mu \mathrm{m}$ devices generally show higher resolutions than $10 \mu \mathrm{m}$ devices. Devices with relatively shorter channels may achieve higher resolutions. Third, the number of SWNT layers can be adjusted. Recent study shows that SWNTs can dramatically enhance the performance of biosensors due to their remarkable charge transfer capability [11]. For our sensor, the interconnection between the SWNT layers provides a highly conductive path for the charge carriers. The increased number of assembled SWNT layers can enhance the conductivity of the thin film and improve the performance of the device. Last, in order to reduce the transient conductance change and increase the stability of the thin film, an additional polyelectrolyte multilayer can be deposited on the device for reinforcement and protection.

\section{Conclusions}

In conclusion, we have fabricated a high-performance ACh sensor based on the nano-assembled SWNT conducting film and AChE sensing film. The LbL nano self-assembly process provides a simple and effective technique to immobilize SWNTs and AChE molecules at room temperature. The assembled AChE molecules catalyze ACh and release hydrogen ions, which can increase the conductance of the SWNT thin film. The SWNT thin film based ACh sensor illustrates a high resolution and a short response time. The performance of the sensor can be further improved by optimizing the design parameters including the channel length, the number of SWNT and AChE layers, and the addition of a polyelectrolyte protection multilayer. Furthermore, with different enzymes immobilized on the surface, the sensor is capable of detecting different types of biomolecules. The combination of the SWNT thin films and the LbL nano self-assembly technique provides a promising method to fabricate inexpensive and high-performance micro/nanoscale biosensors.

\section{Acknowledgements}

This work was partially supported by DARPA MEMS/NEMS Fundamental Research Program through the MF3 Center. We thank Dr. Stephen A. Campbell at the Department of Electrical and Computer Engineering at the University of Minnesota for using his laboratory with the electrical characterization, appreciate $\mathrm{Dr}$. Yi Liu at the Department of Mechanical Engineering at the University of Minnesota for the helpful discussion, also acknowledge the Nanofabrication Center at the University of Minnesota for the help with the fabrication processes.

\section{References}

[1] B. Liu, Y.-H. Yang, Z.-Y. Wu, H. Wang, G.-L. Shen, R.-Q. Yu, A potentiometric acetylcholinesterase biosensor based on plasma-polymerized film, Sensor. Actuat. B-Chem. 104 (2005) 186-190.

[2] H. Varoqui, J.D. Erickson, Active transport of acetylcholine by the human vesicular acetylcholine transporter, J. Biol. Chem. 271 (1996) 27229-27232.

[3] S. Lin, C.-C. Liu, T.-C. Chou, Amperometric acetylcholine sensor catalyzed by nickel anode electrode, Biosens. Bioelectron. 20 (2004) 9-14. 
[4] P. Uutela, R. Reinilä, P. Piepponen, R.A. Ketola, R. Kostiainen, Analysis of acetylcholine and choline in microdialysis samples by liquid chromatography/tandem mass spectrometry, Rapid Commun. Mass Sp. 19 (2005) 2950-2956.

[5] L. Alfonta, E. Katz, I. Willner, Sensing of acetylcholine by a tricomponent-enzyme layered electrode using faradaic impedance spectroscopy, cyclic voltammetry, and microgravimetric quartz crystal microbalance transduction methods, Anal. Chem. 72 (2000) 927-935.

[6] A.B. Kharitonov, M. Zayats, A. Lichtenstein, E. Katz, I. Willner, Enzyme monolayerfunctionalized field-effect transistors for biosensor applications, Sensor. Actuat. B-Chem. 70 (2000) 222-231.

[7] J. Kong, N.R. Franklin, C. Zhou, M.G. Chapline, S. Peng, K. Cho, H. Dai, Nanotube molecular wires as chemical sensors, Science 287 (2000) 622-625.

[8] J. Kong, M.G. Chapline, H. Dai, Functionalized carbon nanotubes for molecular hydrogen sensors, Adv. Mater. 13 (2001) 1384-1386.

[9] K. Besteman, J.-O. Lee, F.G.M. Wiertz, H.A. Heering, C. Dekker, Enzyme-coated carbon nanotubes as single-molecule biosensors, Nano Lett. 3 (2003) 727-730.

[10] K.-S. Teh, L. Lin, MEMS sensor material based on polypyrrole-carbon nanotube nanocomposite: film deposition and characterization, J. Micromech. Microeng. 15 (2005) 2019-2027.

[11] J. Wang, M. Musameh, Carbon nanotube/Teflon composite electrochemical sensors and biosensors, Anal. Chem. 75 (2003) 2075-2079.

[12] W. Xue, T. Cui, Characterization of layer-by-layer self-assembled carbon nanotube multilayer thin films, Nanotechnology 18 (2007) 145709.

[13] W. Xue. T. Cui, A high-resolution amperometric acetylcholine sensor based on nanoassembled carbon nanotubes, IEEE Int. Conf. on Micro Electro Mechanical Systems (Kobe, Japan 2007) 529-532.

[14] F. Hua, J. Shi, Y. Lvov, T. Cui, Patterning of layer-by-layer self-assembled multiple types of nanoparticle thin films by lithographic technique, Nano Lett. 2 (2002) 1219-1222.

[15] R. Martel, T. Schmidt, H.R. Shea, T. Hertel, Ph. Avouris, Single- and multi-wall carbon nanotube field-effect transistors, Appl. Phys. Lett. 73 (1998) 2447-2449.

[16] W. Xue, Y. Liu, T. Cui, High-mobility transistors based on nanoassembled carbon nanotube semiconducting layer and $\mathrm{SiO}_{2}$ nanoparticle dielectric layer, Appl. Phys. Lett. 89 (2006) 163512.

[17] J. L. Alessandrini, J. Vila, Conformation of a single polyelectrolyte chain in the Coulombic unscreened limit, Phys. Rev. E 49 (1994) R3584-R3586. 